\title{
Phosphatidylinositol 4-phosphate 5-kinase $\alpha$ is induced in ganglioside-stimulated brain astrocytes and contributes to inflammatory responses
}

\author{
Sang Yoon Lee ${ }^{1,2,5}$, Bokyung Kim ${ }^{1,2}$, Sarah Yoon ${ }^{3}$, \\ Yeon Joo Kim ${ }^{2}$, Tian Liu ${ }^{1,2}$, Joo Hong Woo, ${ }^{2,4}$, \\ Yong-Joon Chwae ${ }^{3}$, Eun-hye Joe ${ }^{1,4}$ and Ilo Jou ${ }^{2,4}$ \\ ${ }^{1}$ Neuroscience Graduate Program \\ ${ }^{2}$ Chronic Inflammatory Disease Research Center \\ ${ }^{3}$ Department of Microbiology \\ ${ }^{4}$ Department of Pharmacology \\ Ajou University School of Medicine \\ Suwon 443-721, Korea \\ ${ }^{5}$ Corresponding author: Tel, 82-31-219-4408; \\ Fax, 82-31-219-5069; E-mail, sangyoon@ajou.ac.kr \\ DOI 10.3858/emm.2010.42.9.066
}

Accepted 18 August 2010

Available Online 19 August 2010

Abbreviations: EmGFP, emerald green fluorescent protein; miRNA, microRNA; $\mathrm{PI}(4,5) \mathrm{P}_{2}$, phosphatidylinositol 4,5-bisphosphate; PIP5K, phosphatidylinositol 4-phosphate 5-kinase; YFP, yellow fluorescent protein

\begin{abstract}
In brain tissue, astrocytes play defensive roles in central nervous system integrity by mediating immune responses against pathological conditions. Type I phosphatidylinositol 4-phosphate 5-kinase $\alpha$ (PIP5K $\alpha$ ) that is responsible for production of phosphatidylinositol 4,5-bisphosphate $\left(\mathrm{PI}[4,5] \mathrm{P}_{2}\right)$ regulates many important cell functions at the cell surface. Here, we have examined whether PIP5K $\alpha$ is associated with astrocyte inflammatory responses. Gangliosides are releasable from damaged cell membranes of neurons and capable of inducing inflammatory responses. We found that treatment of primary cultured astrocytes with gangliosides significantly enhanced PIP5K $\alpha$ mRNA and protein expression levels. $\mathrm{PI}(4,5) \mathrm{P}_{2}$ imaging using a fluorescent tubby $(\mathbf{R} 332 \mathrm{H})$ expression as a $\mathrm{PI}(4,5) \mathrm{P}_{2}$-specific probe showed that ganglioside treatment increased $\mathrm{PI}(4,5) \mathrm{P}_{2}$ level. Interestingly, microRNA-based PIP5K $\alpha$ knockdown strongly reduced ganglioside-induced transcription of proinflammatory cytokines IL-1 $\beta$ and TNF $\alpha$. PIP5K $\alpha$ knockdown also suppressed ganglioside-induced phosphorylation and nuclear translocation of NF-KB and the
\end{abstract}

degradation of IKB- $\alpha$, indicating that PIP5K $\alpha$ knockdown interfered with the ganglioside-activated NF- $\kappa B$ signaling. Together, these results suggest that PIP5K $\alpha$ is a novel inflammatory mediator that undergoes upregulation and contributes to immune responses by facilitating NF-KB activation in ganglioside-stimulated astrocytes.

Keywords: astrocytes; brain; gangliosides; inflammation; NF-кB; 1-phosphatidylinositol-4-phosphate 5-kinase

\section{Introduction}

Astrocytes are the most abundant glial cells in the brain and play crucial roles as immune effector cells against brain insult (Farina et al., 2007). Reactive astrocytes produce inflammatory mediators, including proinflammatory cytokines such as $\mathrm{IL}-1 \beta$ and TNF $\alpha$, reactive oxygen species, and nitric oxide (Pawate et al., 2004; Jou et al., 2006; Farina et al., 2007). Under pathological conditions such as neurodegenerative disorders, astrocyte immune function exerts a neuroprotective role, but is also recognized to cause the progression of neuronal damage (Teismann and Schulz, 2004; Schwab and McGeer, 2008).

Phosphatidylinositol 4,5-bisphosphate $\left(\mathrm{PI}[4,5] \mathrm{P}_{2}\right)$, generated from phosphatidylinositol 4-phosphate by type I phosphatidylinositol 4-phosphate 5-kinase (PIP5K) family members and also from phosphatidylinositol 5-phosphate by type II phosphatidylinositol 5-phosphate 4-kinase (PIP4K) family members, is enriched in the plasma membrane (Rameh et al., 1997; Doughman et al., 2003). Synthesis of $\mathrm{PI}(4,5) \mathrm{P}_{2}$ is catalyzed mainly by type I PIP5K because phosphatidylinositol 4-phosphate is more abundant than phosphatidylinositol 5-phosphate in mammalian cells (Di Paolo and De Camilli, 2006). PIP5K and $\mathrm{PI}(4,5) \mathrm{P}_{2}$ are critical regulators of a variety of cellular processes involving membrane signaling and trafficking, and actin dynamics (Takenawa and Itoh, 2001; Doughman et al., 2003; Di Paolo and De Camilli, 2006).

Gangliosides, sialic acid-containing glycosphingolipids, are enriched in neuronal cell membranes and released into the extracellular space upon 
A

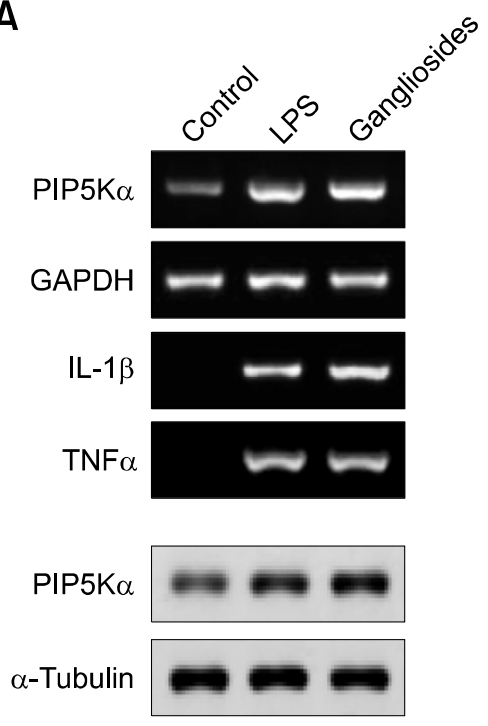

B

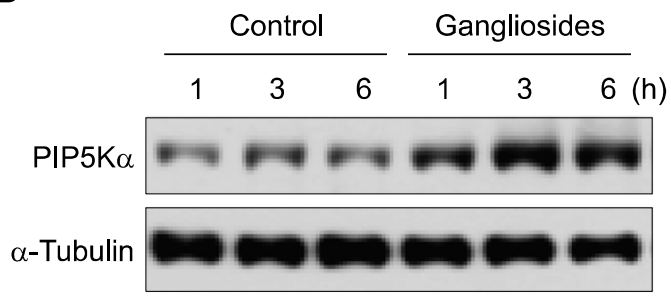

C

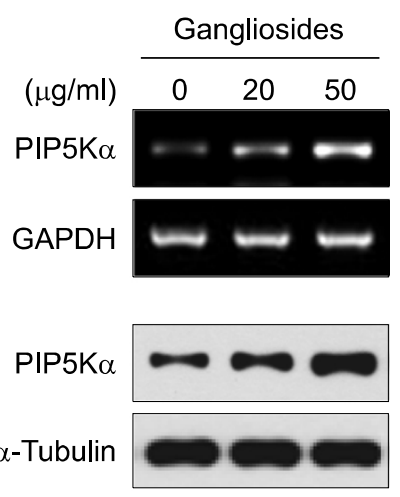

D

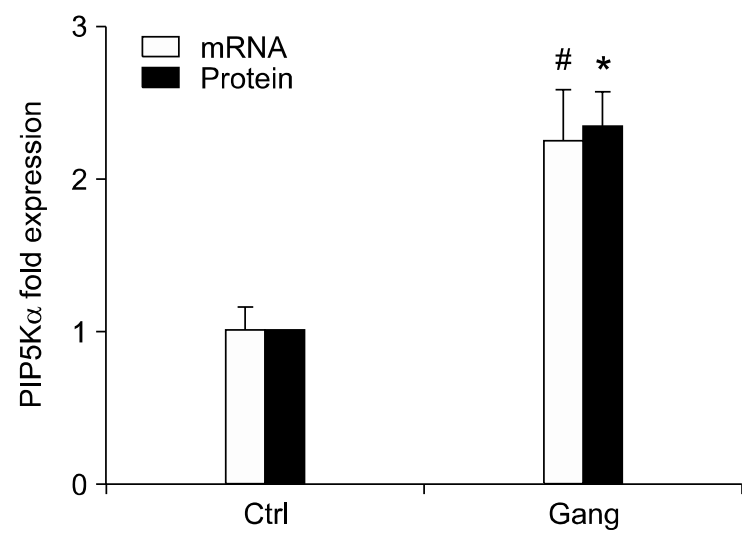

Figure 1. Effects of gangliosides on PIP5K $\alpha$ expression in primary astrocytes. Primary cultured rat astrocytes were serum-starved overnight before treatment. Primary astrocytes were incubated in the presence or absence of $100 \mathrm{ng} / \mathrm{ml} \mathrm{LPS}$ or $50 \mu \mathrm{g} / \mathrm{ml}$ gangliosides for $3 \mathrm{~h}(\mathrm{~A}), 50 \mu \mathrm{g} / \mathrm{ml}$ gangliosides for the indicated times (B), or the indicated concentrations of gangliosides for $3 \mathrm{~h}(C)$. Changes in the mRNA expression levels of IL-1 $\beta$ and TNF $\alpha$ (A), and PIP5K $\alpha(A, C)$ were analyzed by RT-PCR. Changes in the protein expression levels of PIP5K $\alpha(A-C)$ were assayed by Western blot analysis. As a loading control for RT-PCR and Western blot analysis, GAPDH (A, C) and $\alpha$-tubulin (A-C), respectively, were included. (D) After stimulation with $50 \mu \mathrm{g} / \mathrm{ml}$ gangliosides (Gang) for $3 \mathrm{~h}$, PIP5K $\alpha$ mRNA levels were determined by qRT-PCR. Under the same condition, PIP5K $\alpha$ protein levels in (A-C) were measured. Both expression levels were quantified as fold-expression over the unstimulated control (Ctrl). Values are presented as mean $\pm S E M$. ${ }^{*} P<$ 0.01 and ${ }^{\#}<0.05$ compared with the control.

brain injury and play a significant role in various molecular events related to brain pathology and physiology (Blennow et al., 1992; Sonnino and Chigorno, 2000; Ariga et al., 2008; Posse de Chaves and Sipione, 2010). Recently, gangliosides were shown to affect proliferation of mouse embryonic stem cells and differentiation of neural precursor cells (Jung et al., 2009). Furthermore, treatment of astrocytes and microglia (another important glial cell type that mediates brain inflammation) with gangliosides stimulates the activation of multiple signaling proteins, such as NADPH oxidase, PKC, and p42/44 MAPK, and the transcription factor NF- $\mathrm{KB}$, which are major participants in glial inflammatory responses (Pyo et al., 1999; Min et al., 2004; Jou et al., 2006).
Here, we investigated the potential link between PIP5K $\alpha$ (an isoform of PIP5K) and ganglioside-induced astrocyte inflammatory responses. Our findings revealed that gangliosides enhanced PIP5K $\alpha$ expression, which led to an increase in $\mathrm{PI}(4,5) \mathrm{P}_{2}$, and that gene knockdown of PIP5K $\alpha$ attenuated the ganglioside-induced activation of $\mathrm{NF}-\mathrm{kB}$ signaling and inflammatory responses in rat primary cultured astrocytes.

\section{Results}

\section{Ganglioside treatment of primary astrocytes enhances PIP5K $\alpha$ expression}

As a first step to examine whether PIP5K $\alpha$ is 
Tubby-cYFP-R332H fluorescence
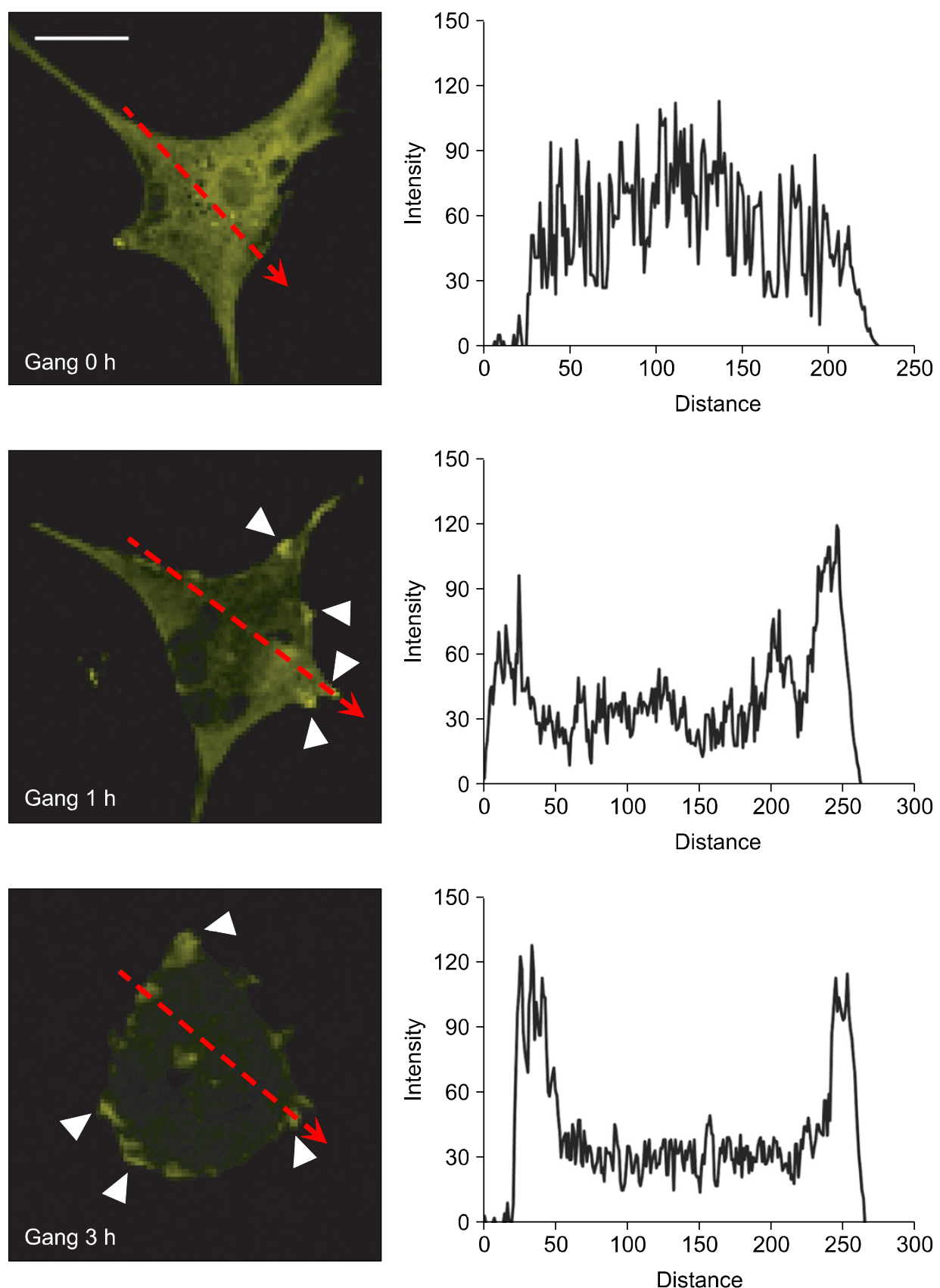

Figure 2. Monitoring changes in $\mathrm{PI}(4,5) \mathrm{P}_{2}$ levels induced by gangliosides in primary astrocytes. Primary astrocytes were transfected with a tubby-cYFP-R332H expression construct, a $\mathrm{PI}(4,5) \mathrm{P}_{2}$-specific probe, using Amaxa Nucleofection. At $48 \mathrm{~h}$ posttransfection, cells were serum-starved and treated with 50 $\mu \mathrm{g} / \mathrm{ml}$ gangliosides (Gang) for 0,1 , or $3 \mathrm{~h}$. YFP fluorescence in the FITC channel was visualized using an LSM 710 confocal microscopy. Arrowheads indicate the localization of the tubby protein in the plasma membrane. Scale bar, $20 \mu \mathrm{m}$. YFP fluorescence intensities along the dotted line arrows in the cell images were analyzed using the Zeiss ZEN 2009 software. Their intensity profiles in the graphs show the translocation of tubby protein between the membrane and the cytosol.

involved in ganglioside-induced astrocyte inflammatory responses, we analyzed PIP5K $\alpha$ expression in primary culture of rat astrocytes. PIP5K $\alpha$ mRNA (Figures $1 \mathrm{~A}$ and $1 \mathrm{C}$ ) and protein (Figures $1 \mathrm{~A}-1 \mathrm{C}$ ) expression, determined by RT-PCR and Western blot analysis, respectively, was detectable in primary astrocytes. We next examined whether ganglioside treatment altered PIP5K $\alpha$ expression levels. The expression of PIP5K $\alpha$ mRNA and protein was significantly enhanced after stimulation with gangliosides for $3 \mathrm{~h}$ (Figure 1A). Under the same assay conditions, LPS was similarly effective in inducing the expression of PIP5K $\alpha$ mRNA and protein (Figure 1A). As expected, both inflammatory stimulators induced increases in IL-1 $\beta$ and TNF $\alpha$ transcription (Figure 1A). Upregulation of PIP5K $\alpha$ protein expression occurred within $1 \mathrm{~h}$ after ganglioside stimulation and continued for up to $3 \mathrm{~h}$, followed by a slight decrease at $6 \mathrm{~h}$, while basal PIP5K $\alpha$ protein levels remained almost 
A

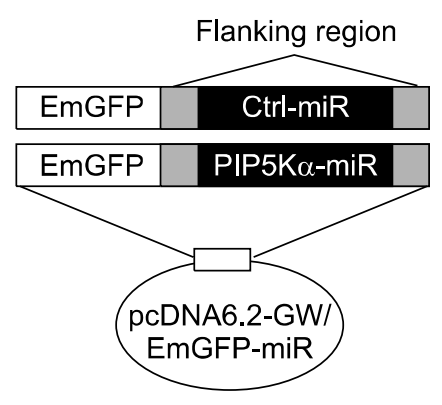

C

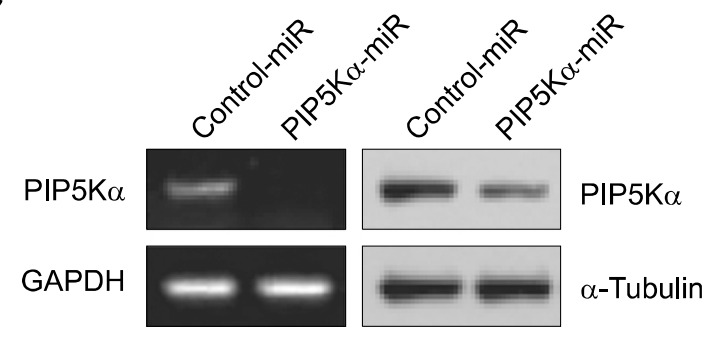

D

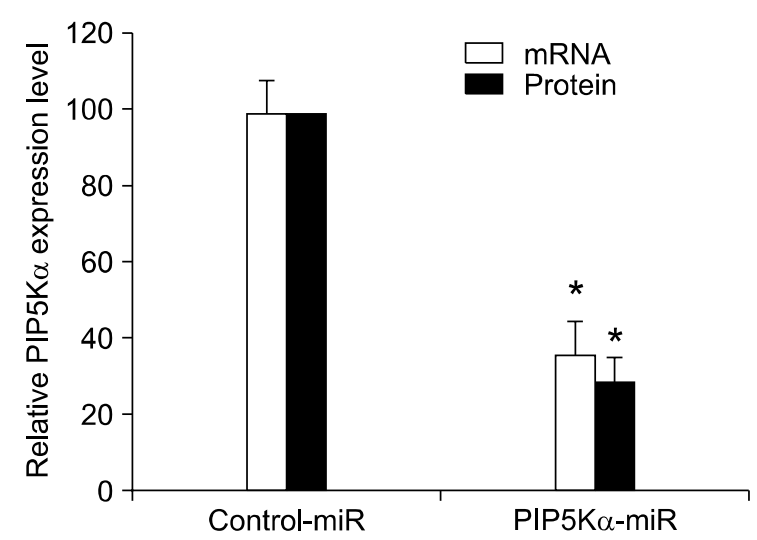

B

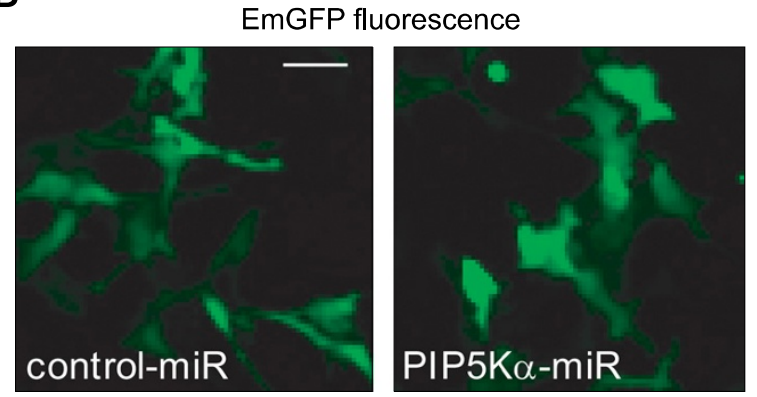

E

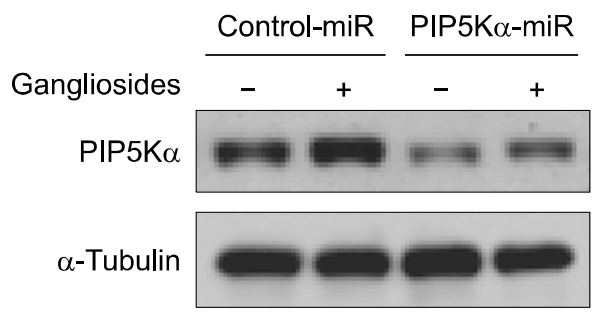

$\mathbf{F}$

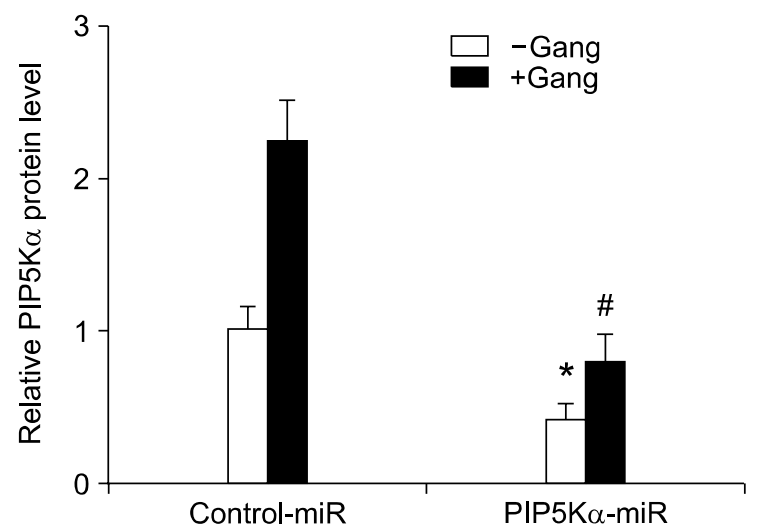

Figure 3. PIP5K $\alpha$ knockdown by miRNA expression and its effect on ganglioside-induced PIP5K $\alpha$ expression. (A) The diagram shows a brief map of

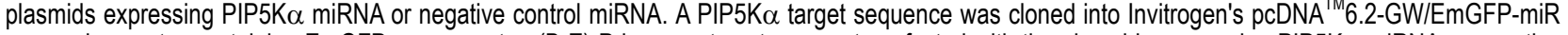
expression vector containing EmGFP as a reporter. (B-E) Primary astrocytes were transfected with the plasmids expressing PIP5K $\alpha$ miRNA or negative control miRNA using Amaxa Nucleofection for $48 \mathrm{~h}$. (B) Cells were visualized by fluorescence microscopy for the detection of EmGFP. Scale bar, $50 \mu \mathrm{m}$. (C) PIP5K $\alpha$ mRNA and protein expression levels were examined by RT-PCR and Western blot analysis, respectively. GAPDH or $\alpha$-tubulin was included as a corresponding loading control. (D) PIP5K $\alpha$ mRNA levels were determined by qRT-PCR analysis and PIP5K $\alpha$ protein levels in (C) were measured. PIP5K $\alpha$ mRNA and protein expression levels in the PIP5K $\alpha$ miRNA were quantified as a percentage of those in negative control miRNA. Values are mean \pm SEM. ${ }^{*} P<0.01$ compared with the control miRNA. (E) After serum starvation overnight, cells were treated with or without 50 $\mu$ g/ml gangliosides for $3 \mathrm{~h}$. PIP5K $\alpha$ protein expression levels were monitored by Western blotting. (F) The PIP5K $\alpha$ protein levels in (E) were quantified relative to that in unstimulated negative control miRNA. Values are mean \pm SEM. ${ }^{*} P<0.01$ and ${ }^{\sharp} P<0.05$ compared with the unstimulated control miRNA.

constant in the resting conditions (Figure 1B). Gangliosides enhanced the expression of PIP5K $\alpha$ mRNA and protein in a dose-dependent manner (Figure $1 \mathrm{C})$. On average, gangliosides $(50 \mu \mathrm{g} / \mathrm{ml}, 3$ h) increased the mRNA, which was determined by quantitative real-time RT-PCR (qRT-PCR) analysis, and protein expression levels of PIP5K $\alpha$ by appro- ximately 2.3-fold with some quantitative variances among experiments (Figure 1D).

\section{Ganglioside treatment increases $\mathrm{PI}(4,5) \mathrm{P}_{2}$ levels in primary astrocytes}

$\mathrm{PI}(4,5) \mathrm{P}_{2}$ is synthesized mainly by PIP5K. Because 
treatment with gangliosides enhanced PIP5K $\alpha$ expression, we hypothesized that ganglioside stimulation augments the production of $\mathrm{PI}(4,5) \mathrm{P}_{2}$. To test this, we measured $\mathrm{PI}(4,5) \mathrm{P}_{2}$ levels before and after ganglioside treatment by transfection and imaging of the tubby mutant $(\mathrm{R} 332 \mathrm{H})$ fused to yellow fluorescent protein (YFP) at the C-terminus (tubby-cYFP-R332H) (Quinn et al., 2008). The $\mathrm{C}$-terminal tubby domain of the transcription factor tubby specifically binds to plasma membrane $\mathrm{Pl}(4,5) \mathrm{P}_{2}$ (Santagata et al., 2001). Fluorescent tubby constructs such as tubby-cYFP-R332H have been used to monitor changes in the $\mathrm{PI}(4,5) \mathrm{P}_{2}$ level that are reflected by differences in relative tubby fluorescence intensities between the plasma membrane and the cytoplasm (Santagata et al., 2001; Quinn et al., 2008; Szentpetery et al., 2009). Confocal images of the expressed tubby-cYFP. $\mathrm{R} 332 \mathrm{H}$ fusion protein showed a diffuse pattern of YFP signals throughout the cytoplasm in the resting condition (zero-time) (Figure 2). After 1 or 3 h stimulation with gangliosides, YFP signals in the plasma membrane increased and YFP signals in the cytoplasm decreased in a time-dependent manner. The changed intensity profiles obtained from image analysis indicated that gangliosides triggered $\mathrm{PI}(4,5) \mathrm{P}_{2}$ production in the plasma membrane (Figure 2).

\section{PIP5K $\alpha$ knockdown attenuates ganglioside-induced inflammatory responses in primary astrocytes}

Because PIP5K $\alpha$ and $\mathrm{PI}(4,5) \mathrm{P}_{2}$ are important regulators of diverse cellular functions (Doughman et al., 2003; Di Paolo and De Camilli, 2006), we investigated whether PIP5K $\alpha$ is involved in regulating ganglioside-induced inflammatory responses. To explore this possibility, we first developed a method of PIP5K $\alpha$ knockdown using a vector-based microRNA (miRNA) expression system. Complementary oligonucleotides harboring the PIP5K $\alpha$ target sequence $(1,844-1,864 \mathrm{bp})$ were cloned into a pcDNA ${ }^{7 \mathrm{M}} 6.2-\mathrm{GW} / \mathrm{EmGFP}-\mathrm{miR}$ expression vector that contains Emerald green fluorescent protein (EmGFP), a variant of enhanced GFP, as a reporter (Figure 3A). This construct was efficiently expressed in primary astrocytes by Amaxa Nucleofection, as assessed by the presence of EmGFP $(\sim 70 \%)$ in fluorescence microscopy (Figure 3B). As a negative control that does not target mammalian genes, a pcDNA ${ }^{\mathrm{TM}}$ 6.2-GW/EmGFP-miR-neg control plasmid, which was supplied by the manufacturer, was expressed in the same manner (Figures $3 \mathrm{~A}$ and 3B). qRT-PCR and Western blot analysis demonstrated that the expression of PIP5K $\alpha$ miRNA significantly reduced PIP5K $\alpha$ mRNA
(27-45\%) and protein (21-36\%) levels compared to the expression levels (set as 100\%) produced by the control miRNA (Figures $3 \mathrm{C}$ and 3D). As expected, ganglioside-induced upregulation of PIP5K $\alpha$ protein levels was observed in negative control cells (Figures $3 \mathrm{E}$ and $3 \mathrm{~F}$ ). In contrast, gangliosides did not substantially upregulate PIP5K $\alpha$ protein levels in PIP5K $\alpha$-knockdown cells (Figures 3E and 3F).

We next investigated whether the expression of PIP5K $\alpha$ miRNA affected astrocyte immune function by comparing the time courses of ganglioside-induced transcriptional changes in PIP5K $\alpha$, IL-1 $\beta$, TNF $\alpha$, and TGF $\beta$ between negative control cells and PIP5K $\alpha-k n o c k d o w n$ cells using RT-PCR and qRT-PCR analysis. Transcriptional increases in PIP5K $\alpha$ induced by gangliosides occurred time-dependently for up to $3 \mathrm{~h}$ in negative control cells, but were remarkably weakened in PIP5K $\alpha$-knockdown cells over the same time period (Figures $4 A$ and $4 B$ ). Similarly, IL-1 $\beta$ and TNF $\alpha$ transcription induced by gangliosides were reduced in PIP5K $\alpha$-knockdown cells compared to negative control cells at all time points examined (Figures $4 \mathrm{~A}$ and $4 \mathrm{~B}$ ). TGF $\beta$ mRNA levels were not affected by gangliosides in negative control cells and were also unaffected by PIP5K $\alpha$ knockdown (Figures 4A and 4B). These results indicate that PIP5K $\alpha$ knockdown exerts inhibitory effects on astrocyte inflammatory responses that are stimulated by gangliosides.

\section{PIP5K $\alpha$ knockdown interferes with ganglioside-activated NF- $\kappa$ B signaling in primary astrocytes}

$\mathrm{NF}-\kappa \mathrm{B}$ is a major transcription factor responsible for the induction of proinflammatory cytokines in response to inflammatory stimuli in various types of immune cells ( $\mathrm{Li}$ and Verma, 2002). The strong inhibition of PIP5K $\alpha$ knockdown on the stimulating effects of gangliosides on IL-1 $\beta$ and TNF $\alpha$ transcription (Figure 4) suggested that PIP5K $\alpha$ knockdown might interfere with ganglioside-triggered $N F-\kappa B$ activation. Activation of the $N F-\kappa B$ signaling pathway involves the degradation of $I \kappa B$ through the ubiquitin-proteasome system, which induces nuclear translocation of $\mathrm{NF}-\kappa \mathrm{B}$ ( $\mathrm{Li}$ and Verma, 2002). In addition, the serine 536 residue in the $N F-\kappa B$ p65 (a member of the NF- $\kappa B$ family) transactivation domain is phosphorylated by $I_{\kappa} B$ kinase $\beta$ in response to LPS or TNF $\alpha$, leading to its transcriptional activity (Sakurai et al., 1999; Yang et al., 2003). We tested whether PIP5K $\alpha$ knockdown perturbs such molecular changes in $1 \kappa B$ and $N F-\kappa B$ p65 induced by gangliosides in primary 


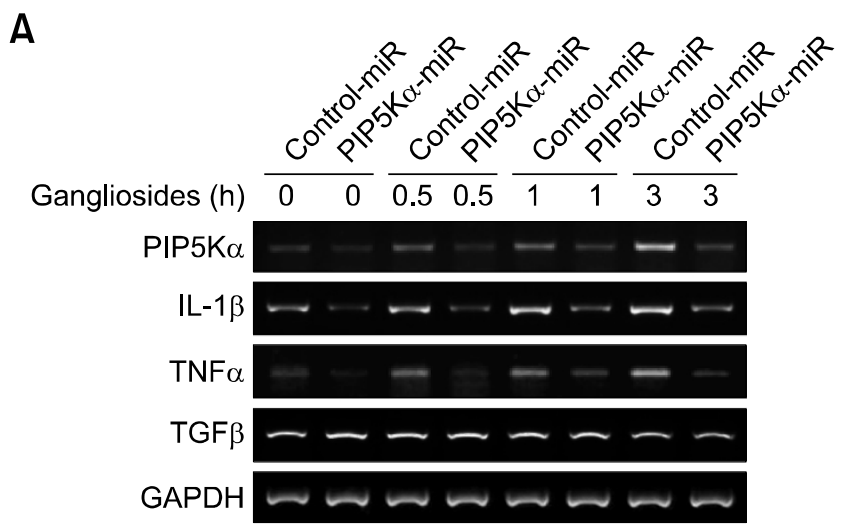

B
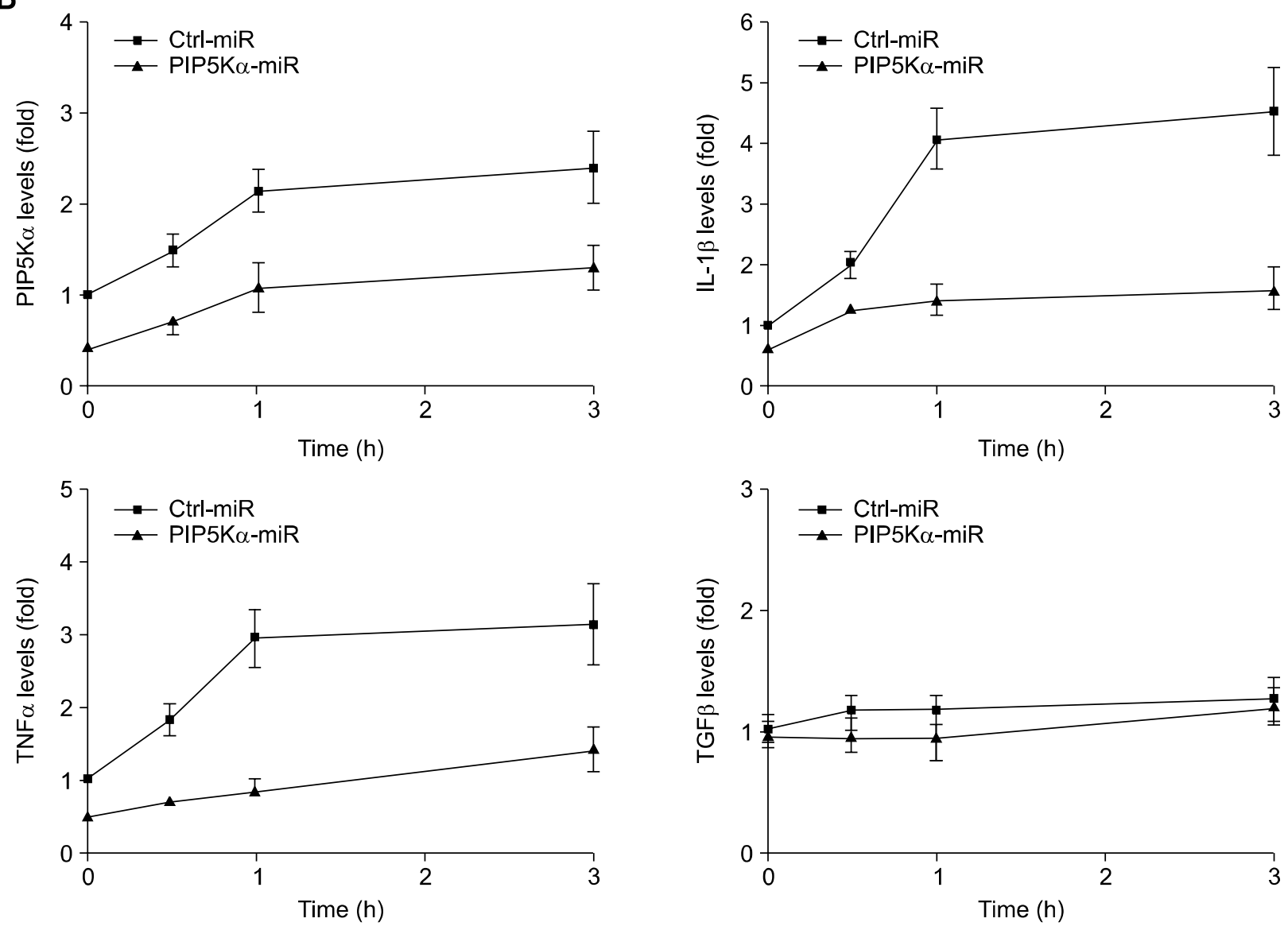

Figure 4. Effects of PIP5K $\alpha$ knockdown on ganglioside-induced transcription of inflammatory cytokines. (A, B) Primary astrocytes were transfected with PIP5K $\alpha$ miRNA or negative control miRNA expression plasmids using Amaxa Nucleofection for $48 \mathrm{~h}$. Following serum starvation overnight, cells were incubated in the presence or absence of $50 \mu \mathrm{g} / \mathrm{ml}$ gangliosides for the indicated times. (A) mRNA expression levels of PIP5K $\alpha$, IL-1 $\beta$, TNF $\alpha$, TGF $\alpha$, and GAPDH (a loading control) were assayed by RT-PCR analysis. (B) The time-dependent transcriptional changes in each gene were analyzed by qRT-PCR and quantified as fold-expression over the negative control miRNA at the zero-time.

astrocytes. Stimulation of negative control cells with gangliosides for 15 min induced both the degradation of $I_{\kappa} B-\alpha$ and the phosphorylation of NF-kB p65 at serine 536, compared to the unstimulated condition (Figures $5 A$ and $5 B$ ). In contrast, in PIP5K $\alpha$-knockdown cells, IкB- $\alpha$ levels remained relatively high and NF-KB p65 remained unphosphorylated (Figures $5 \mathrm{~A}$ and $5 \mathrm{~B}$ ).

Under the same assay conditions, we examined the subcellular localization of NF-kB p65 immuno- 
A

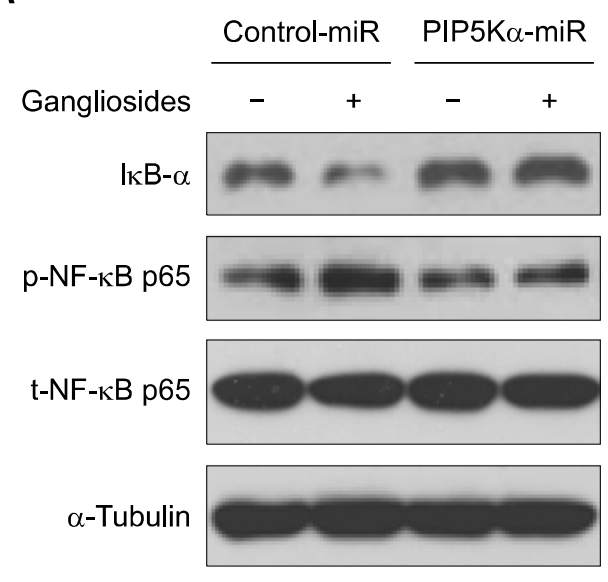

B
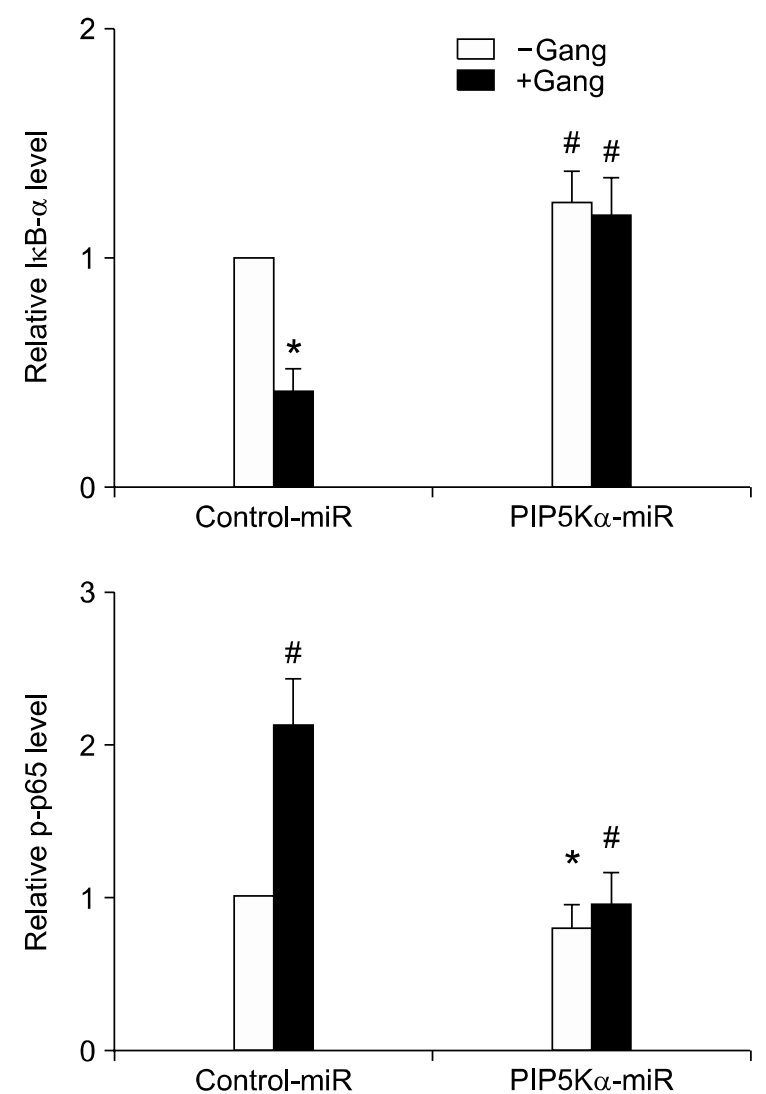

Figure 5. Effects of PIP5K $\alpha$ knockdown on ganglioside-stimulated NF-KB signaling. (A, C) Primary astrocytes were transfected with the miRNA expression plasmids and treated as described above. (A) After treatment with or without $50 \mu \mathrm{g} / \mathrm{ml}$ gangliosides for $15 \mathrm{~min}$, cell lysates were prepared and changes in protein levels of $I_{\kappa} B-\alpha$, phosphorylated and total NF- $\kappa B$ p65, and $\alpha$-tubulin (a loading control) were examined by Western blot analysis using their specific antibodies. (B) $\mid \kappa B-\alpha$ and phosphorylated NF- $\kappa B$ p65 levels in $(A)$ were quantified relative to those in unstimulated negative control miRNA. Values are mean \pm SEM. ${ }^{*} P<0.01$ and ${ }^{\#} P<0.05$ compared with the unstimulated control miRNA. (C) Cells were stimulated with gangliosides under the same condition as $(A)$ and then immunostained with a specific primary antibody against NF- $\kappa B$ p65, followed by an Alexa Fluor 594 -conjugated secondary antibody. The transfected cells and the nuclei were visualized by EmGFP and DAPI staining, respectively. Cell images were obtained by an LSM 710 confocal microscopy under the laser filter sets (FITC, Rhodamine, and DAPI). Scale bar, $20 \mu \mathrm{m}$.

cytochemically using an anti-NF-кB p65 antibody. Confocal images revealed that NF- $\mathrm{KB}$ p65 was distributed in the cytosol in both unstimulated negative control and PIP5K $\alpha$-knockdown cells (Figure 5C). The ganglioside-dependent nuclear translocation of NF-kB p65 was observed in negative control cells, but not in PIP5K $\alpha$-knockdown cells (Figure $5 \mathrm{C}$ ), consistent with the results of the Western blot analysis (Figure 5A). Together, these results indicate that PIP5K $\alpha$ positively regulates the ganglioside-induced activation of NF- $\mathrm{kB}$ signaling pathway.

\section{Discussion}

Accumulating evidence indicates that the PIP5K-driven production of $\mathrm{PI}(4,5) \mathrm{P}_{2}$ regulates signal trans- duction, membrane trafficking, actin cytoskeletal reorganization, and ion channel modulation (Takenawa and Itoh, 2001; Suh and Hille, 2005; Di Paolo and De Camilli, 2006). Despite the essential pleiotropic functions of $\mathrm{PIP5K}$ and $\mathrm{PI}(4,5) \mathrm{P}_{2}$, however, little is known about their roles in glial inflammatory responses in the brain. The findings of the present study indicate that PIP5K $\alpha$ undergoes transcriptional and translational upregulation in response to gangliosides in brain astrocytes. The concomitant induction of IL- $1 \beta$ and TNF $\alpha$ by gangliosides provides novel evidence that PIP5K $\alpha$ is an inflammatory mediator that participates in astrocyte inflammatory responses. $\mathrm{PI}(4,5) \mathrm{P}_{2}$ level is regulated by alterations in expression level or catalytic activity of PIP5K. PIP5K-mediated $\mathrm{PI}(4,5) \mathrm{P}_{2}$ synthesis is achieved by changes in the catalytic activity of PIP5K through various 
C
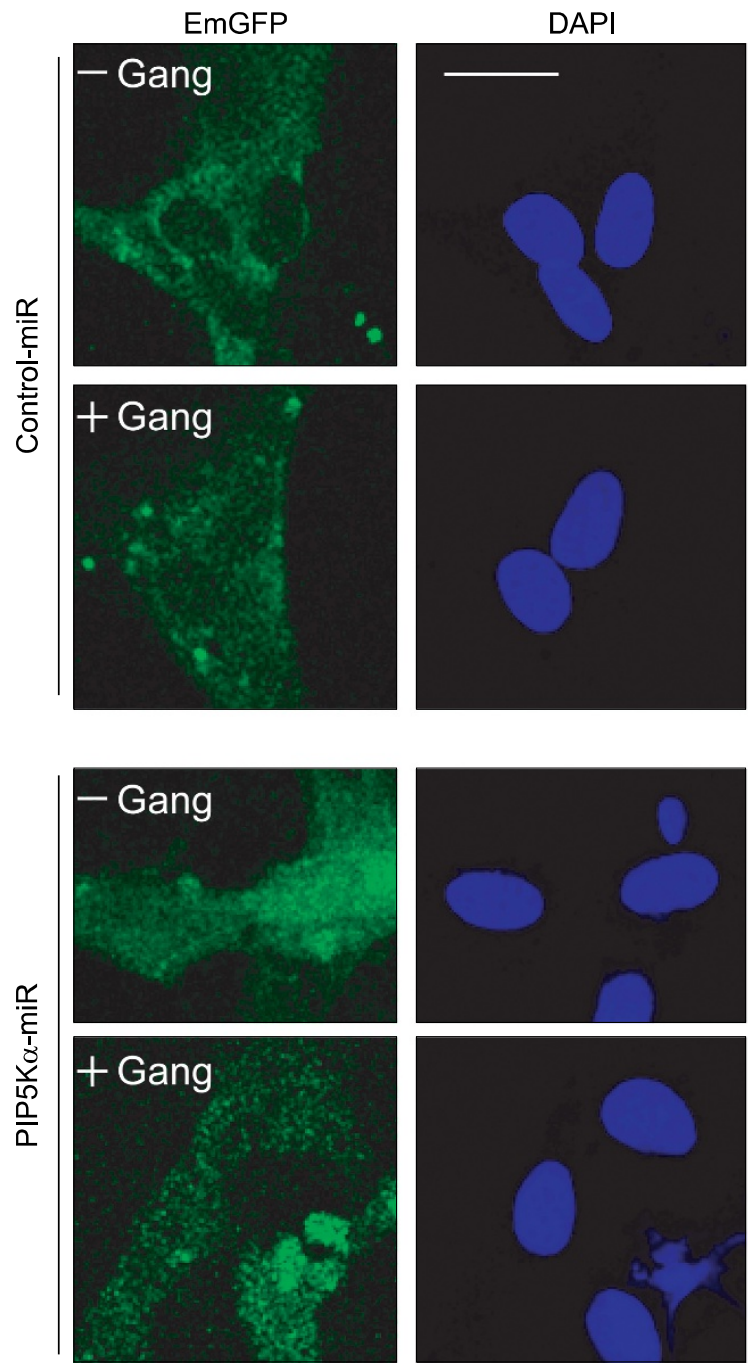
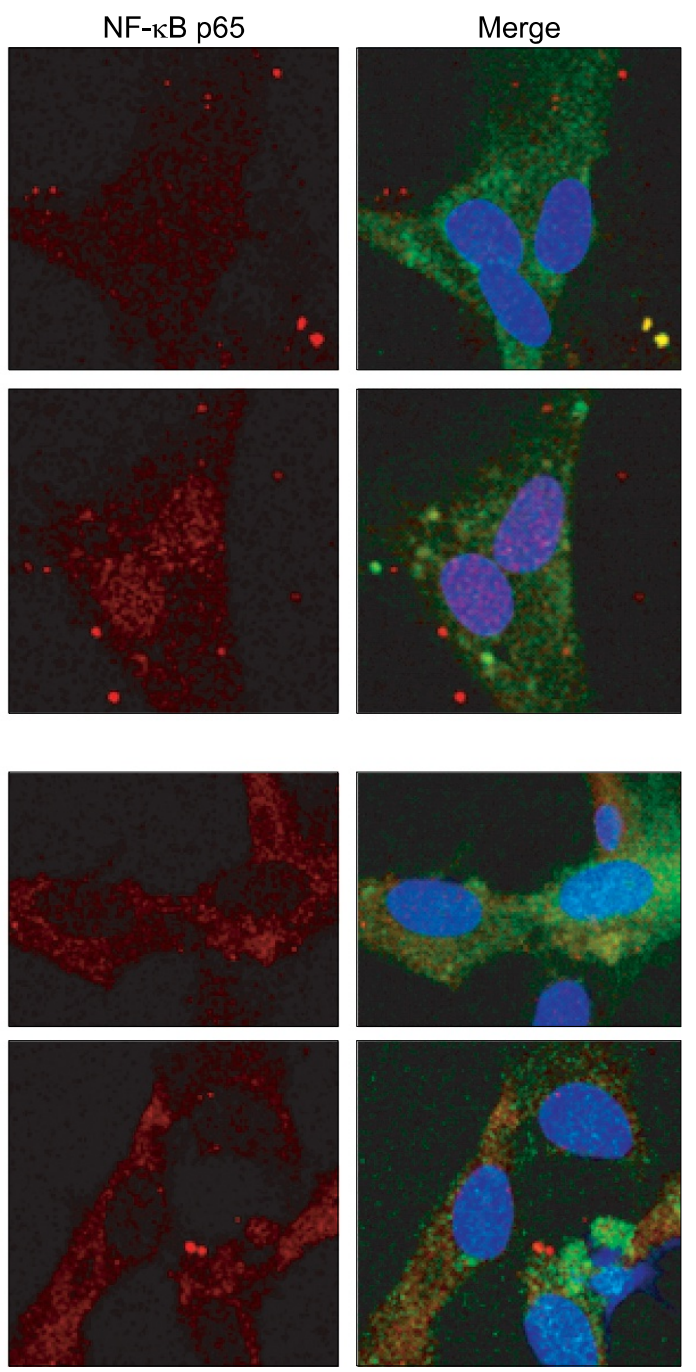

Figure 5. Continued.

regulatory mechanisms, such as protein-protein interactions and protein phosphorylation (van den Bout and Divecha, 2009). $\mathrm{Pl}(4,5) \mathrm{P}_{2}$ levels are also under the control of changes in the PIP5K expression level, as demonstrated by the effects of gene knockdown or overexpression of PIP5K as a means of manipulating $\mathrm{PI}(4,5) \mathrm{P}_{2}$ levels (Yamamoto et al., 2001; Padron et al., 2003). In addition, inflammatory responses are accompanied by extensive changes in metabolic pathways, based on the induction of gene expression and the production of inflammatory mediators. Hence, the increase in $\mathrm{PI}(4,5) \mathrm{P}_{2}$ induced by gangliosides is, at least in part, likely due to the upregulation of PIP5K $\alpha$ expression, although we cannot at this time exclude the possibility that stimulation of PIP5K by gangliosides increases $\mathrm{PI}(4,5) \mathrm{P}_{2}$.
$\mathrm{PI}(4,5) \mathrm{P}_{2}$ levels in primary astrocytes were evaluated by $\mathrm{PI}(4,5) \mathrm{P}_{2}$ imaging with an expression construct of tubby-cYFP-R332H. The transcription factor tubby localizes to the plasma membrane due to its interaction with $\mathrm{PI}(4,5) \mathrm{P}_{2}$ (Santagata et al., 2001). Following ligand stimulation of G-protein-coupled receptors, activated phospholipase C- $\beta$ degrades $\mathrm{PI}(4,5) \mathrm{P}_{2}$ and the resulting decrease in $\mathrm{PI}(4,5) \mathrm{P}_{2}$ releases tubby from the plasma membrane to cytoplasm and nucleus (Santagata et al., 2001). The expression of tubby-cYFP-R332H sensitively reflected the $\mathrm{PI}(4,5) \mathrm{P}_{2}$-dependent tubby translocation (Quinn et al., 2008). Taking advantage of this property of the tubby mutant, we found that gangliosides induced the translocation of tubby-cYFP-R332H from the cytoplasm to the plasma membrane. The tubby imaging results 
support that the increased $\mathrm{PI}(4,5) \mathrm{P}_{2}$ production induced by gangliosides occurs specifically at the plasma membrane.

To evaluate the role of ganglioside-induced PIP5K $\alpha$ upregulation in primary astrocytes, we knocked down PIP5K $\alpha$ expression using a miRNA expression system. We demonstrated that PIP5K $\alpha$ knockdown inhibited the gangliosides-induced increase in the transcription of IL-1 $\beta$ and TNF $\alpha$ as well as the ganglioside-induced activation of $\mathrm{NF}-\kappa \mathrm{B}$ signaling. Ganglioside stimulation of primary astrocytes and microglia activates NF- $\mathrm{KB}$ signaling (Pyo et al., 1999; Min et al., 2004; Jou et al., 2006). Thus, it is likely that the attenuated transcription of IL-1 $\beta$ and TNF $\alpha$ in PIP5K $\alpha$-knockdown cells is due to the suppression of NF-KB signaling by PIP5K $\alpha$ knockdown. Taken together, our results suggest that increased PIP5K $\alpha$ expression contributes to ganglioside-induced cytokine production by facilitating the NF- $\mathrm{kB}$ signaling pathway.

Previously, it was reported that Toll-like receptor 4 (TLR4) knockdown by small interfering RNA or transfection of dominant negative TLR4 in primary astrocytes strongly inhibits ganglioside-stimulated $\mathrm{IL}-1 \beta$ and TNF $\alpha$ transcription, NF-KB activation, and IL-6 promoter activity, suggesting an essential role of TLR4 in ganglioside-induced astrocyte inflammatory responses (Jou et al., 2006). Interestingly, $\mathrm{PI}(4,5) \mathrm{P}_{2}$ has an important role in the LPS/TLR4 signaling pathway in bone marrow-derived macrophages (Kagan and Medzhitov, 2006). Upon LPS stimulation, $\mathrm{PI}(4,5) \mathrm{P}_{2}$ levels are increased and subsequently recruit TIRAP (a Toll/IL-1 receptor domain-containing adaptor protein), required for myeloid differentiation factor 88 (MyD88)-dependent TLR4 signaling pathway (Fitzgerald et al., 2001; Horng et al., 2002), to the plasma membrane by mediating its specific interaction with TIRAP (Kagan and Medzhitov, 2006). The resulting $\mathrm{PI}(4,5) \mathrm{P}_{2}$-dependent concentration of TIRAP in the plasma membrane was further suggested to promote formation of the TLR4-TIRAP-MyD88 complex, thereby inducing production of a proinflammatory cytokine IL-6 (Kagan and Medzhitov, 2006). We found that LPS also upregulates PIP5K $\alpha$ expression levels. The TLR4 signaling pathways and downstream effector NF- $\mathrm{KB}$ are well conserved among innate immune cells, including astrocytes (Akira and Takeda, 2004; Farina et al., 2007). Based on the similarities between these previous results and the present findings, PIP5K $\alpha$ may participate in such $\mathrm{PI}(4,5) \mathrm{P}_{2}$-mediated TLR4 signaling in brain astrocytes. Whether $\mathrm{PI}(4,5) \mathrm{P}_{2}$-mediated recruitment of TIRAP to the cell surface TLR4 is involved in inflammatory responses to gangliosides, however, remains to be determined.

While we have focused on PIP5K $\alpha$ in this study, role of other type I PIP5K isoforms, PIP5K $\beta$ and PIP5K $\gamma$, in astrocyte inflammatory responses needs to be examined. PIP5K $\beta$ and/or PIP5K $\gamma$ may play a redundant role in astrocyte immune function. However, PIP5Ks can also be differentially regulated, thereby controlling spatiotemporal pattern of $\mathrm{PI}(4,5) \mathrm{P}_{2}$ production (Mao and Yin, 2007; van den Bout and Divecha, 2009). Mouse PIP5K $\alpha$ deficiency accompanying decrease in $\mathrm{PI}(4,5) \mathrm{P}_{2}$ levels resulted in hypersensitive allergic inflammation and mast cell degranulation upon cross-linking of Fce receptor-I by antigen (Sasaki et al., 2005). Protein expression of mouse PIP5K $\beta$ was elevated in LPS-stimulated BV2 microglial cells (Lund et al., 2006). A plasma membrane $\mathrm{PI}(4,5) \mathrm{P}_{2}$ pool was present in the lipid rafts that are enriched in gangliosides (Szymanska et al., 2009; Posse de Chaves and Sipione, 2010). Upon activation of Fc $\gamma$ receptor IIA in BHK cells, PIP5K $\alpha$ was recruited to the $\mathrm{PI}(4,5) \mathrm{P}_{2}$-rich lipid rafts and colocalized with the receptor, which was suggested to contribute to $\mathrm{Fc} \gamma$ receptor IIA-mediated signaling (Szymanska et al., 2009). These cell type-specific PIP5K responses raise a possibility that PIP5Ks may differentially modulate immune responses in a microenvironment-dependent manner. All three PIP5Ks are expressed in the brain tissue (Ishihara et al., 1996, 1998). Thus, it will be necessary to monitor alterations of in vivo expression and localization of each PIP5K during astrocyte immune responses.

In summary, we report that PIP5K $\alpha$ expression is enhanced in ganglioside-stimulated astrocytes. Our results also suggest that the upregulation of PIP5K $\alpha$ contributes to NF- $\kappa B$ signaling and inflammatory responses that are activated by gangliosides. To our knowledge, these findings are the first demonstration of a potential link between PIP5K $\alpha$ and astrocyte inflammatory responses. As such, this study provides new insight into the roles of PIP5K $\alpha$ and $\mathrm{PI}(4,5) \mathrm{P}_{2}$ in innate immunity mediated by brain astrocytes.

\section{Methods}

\section{Materials}

An ammonium salt form of purified gangliosides was purchased from Matreya (Pleasant Gap, PA). Most research chemicals including LPS and anti- $\alpha$-tubulin antibody were purchased from Sigma (St. Louis, MO). MEM, FBS, and penicillin/streptomycin were obtained from Hyclone (Logan, UT). An anti-PIP5K $\alpha$ antibody was obtained from Santa Cruz Biotechnology (Santa Cruz, CA). 


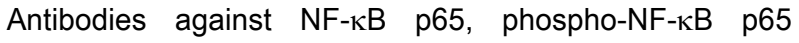
(Ser536), and IKB- $\alpha$ were purchased from Cell Signaling Technology (Beverly, MA).

\section{Cell culture and treatment}

Primary astrocytes were cultured from the cerebral cortices of 1-day-old Sprague-Dawley rats (Samtako, Osan, Korea) as described previously (Min et al., 2004; Jou et al., 2006). Briefly, the cortices were triturated into single cells, plated into T-75 flasks, and grown in MEM containing 10\% FBS and penicillin/streptomycin at $37^{\circ} \mathrm{C}$ in a humidified atmosphere of $5 \% \mathrm{CO}_{2}$ and $95 \%$ air. After culture for 2 weeks, primary microglial cells were removed by mild shaking. Adherent primary astrocytes were washed with PBS, trypsinized, and resuspended in MEM containing 5\% FBS and penicillin/streptomycin. After replating into $60-\mathrm{mm}$ dishes $\left(1 \times 10^{6}\right.$ cells/dish) for 2 days, cells were serum-starved overnight and then treated with gangliosides or LPS under the indicated conditions. The purity of primary cultures was tested by immunostaining for glial fibrillary acidic protein, a marker protein for astrocytes. Confocal microscopy observations confirmed that primary cultures were more than $90 \%$ pure.

\section{Reverse transcription-polymerase chain reaction}

Astrocytes were washed with ice-cold PBS and harvested in a TRIzol lysis reagent (Invitrogen, Carlsbad, CA) and total mRNA was isolated following the supplier's instructions. cDNA was synthesized from isolated total mRNA using avian myeloblastosis virus reverse transcriptase (Takara, Japan) according to the manufacturer's instructions. cDNA was amplified by PCR using the following oligonucleotide primers (Bioneer, Daejeon, Korea): (sense) 5'-CTGAGGGACCTTATGCCTCTC-3' and (antisense) 5'-GAAGAGGGAACCGCTAGCTC-3' for rat PIP5K $\alpha$ (NM_001042621, 546 amino acids); and (sense) 5'-GAGAGCCCTGGATACCAACTACTG-3' and (antisense) 5'-GTGTGTCCAGGCTCCAAATGTAG-3 for rat TGF $\beta$. In this study, the previous mouse and rat PIP5K $\beta$ is referred to as PIP5K $\alpha$, and vice versa, according to the revised nomenclature in the current GenBank database. The primers used to amplify IL-1 $\beta$, TNF $\alpha$, and GAPDH were previously described (Min et al., 2004). Amplified PCR products were separated by electrophoresis on $1.5 \%$ agarose gels and detected under ultraviolet light. Gel images were obtained using the Gel Doc molecular imaging system (Bio-Rad Laboratories, Hercules, CA).

\section{Quantitative real-time PCR}

cDNA was prepared as describe above and qRT-PCR was performed on an ABI PRISM 7000 real-time PCR machine (Applied Biosystems, Foster City, CA) using a SYBR Premix Ex Taq II reagent (Takara, Japan) according to the manufacturer's protocol. The following specific primers (Bioneer) were used: (sense) 5'-TGACTACGAAGGCGGAAGTG-3' and (antisense) 5'-AAACTGGGACCAGGAACAGG-3' for PIP5K $\alpha$; (sense) 5'-TGATGTTCCCATTAGACAGC-3' and (antisense) 5'-GAGGTGCTGATGTACCA-
GTT-3' for IL-1 $\beta$; (sense) 5'-CGTCTACTCCTCAGAGCCCC-3' and (antisense) 5'-TCCACTCAGGCATCGACATT-3' for TNF $\alpha$; and (sense) 5'-GGCCAAAAGGGTCATCATC-3' and (antisense) 5'-GTGATGGCATGGACTGTGG-3' for GAPDH (a housekeeping gene). qRT-PCR primers for TGF $\alpha$ were the same as those described above. Generation of a single gene-specific PCR product was confirmed by melting curve analysis. All PCR reaction samples were prepared in triplicate for each gene. The relative mRNA expression levels were determined by the $2^{-\Delta \Delta} \mathrm{Ct}$ methods $[\Delta \mathrm{Ct}=\mathrm{Ct}$ (target gene) - Ct (GAPDH), $\Delta \Delta \mathrm{Ct}=\Delta \mathrm{Ct}$ (gangliosides) $-\Delta \mathrm{Ct}$ (control) or $\Delta \mathrm{Ct}$ (PIP5K $\alpha$-knockdown cells) - $\Delta \mathrm{Ct}$ (control cells)].

\section{Western blot analysis}

Astrocytes were scraped into a lysis buffer (50 mM Tris $\mathrm{pH}$ 7.4, $150 \mathrm{mM} \mathrm{NaCl}, 1 \mathrm{mM}$ EDTA, $1 \mathrm{mM}$ EGTA, $1 \mathrm{mM}$ dithiothreitol, $1 \mathrm{mM} \mathrm{Na}_{3} \mathrm{VO}_{4}, 5 \mathrm{mM} \mathrm{NaF}$, and $1 \%$ Triton $\mathrm{X}-100)$ containing protease inhibitor cocktail tablets (Roche, Mannheim, Germany) and cell lysates were cleared by centrifugation $\left(15,000 \times \mathrm{g}, 20 \mathrm{~min}, 4^{\circ} \mathrm{C}\right)$. Protein concentration was determined using BCA protein assay reagents (Pierce, Rockford, IL), and 30 to $40 \mu \mathrm{g}$ of cell lysates were separated by SDS-PAGE on $8 \%$ to $10 \%$ resolving gels, and transferred to nitrocellulose membranes (Schleicher \& Schuell Bioscience, Germany). Following blocking with $5 \%$ nonfat milk solution, membrane blots were probed with anti-PIP5K $\alpha$ or anti- $\alpha$-tubulin antibodies, washed four times with Tris-buffered saline containing $0.1 \%$ Tween-20, and further probed with horseradish peroxidase-conjugated secondary antibodies (Zymed Laboratories, San Francisco, CA). Phosphorylated and total $N F-\kappa B \quad p 65$, and $1 \kappa B-\alpha$ were detected using corresponding antibodies following the manufacturer's protocol. The resulting immune complexes were visualized using an enhanced chemiluminescence detection system (Santa Cruz Biotechnology).

\section{PIP5K $\alpha$ knockdown}

We made an expression construct encoding PIP5K $\alpha$ miRNA using BLOCK-iT Pol II miR RNAi Expression Vector Kits (Invitrogen). The following 64-bp oligonucleotides harboring the rat PIP5K $\alpha$ sequences were synthesized (Genotech, Daejeon, Korea): (top strand) 5'-TGCTGAACAGGTGAACCCTCACTTATGTTTTGGCCACTGACTGAC ATAAGTGAGTTCACCTGTT-3', where 21-bp antisense and 19-bp (2 bp removed) sense sequences are indicated by the underlines, respectively, and (bottom strand) 5'-CCTGAACAGGTGAACTCACTTATGTCAGTCAGTGGC CAAAACATAAGTGAGGGTTCACCTGTTC-3'. According to the manufacturers instructions, the single-stranded oligos were annealed and ligated into the pcDNA $^{\mathrm{TM}}$ 6.2-GW/EmGFP-miR expression vector. After transformation, spectinomycin-resistant Escherichia coli clones were isolated and identified by DNA sequencing (Genotech). PIP5K $\alpha$ miRNA expression plasmids were purified using an EndoFree Plasmid Maxi Kit (Qiagen, Hilden, Germany) and transiently transfected into primary astrocytes by Amaxa Nucleofection using a rat astrocyte 
Nucleofector Kit (Amaxa Biosystems, Cologne, Germany) according to the manufacturer's instructions. Briefly, primary astrocytes $\left(2 \times 10^{6}\right.$ cells $)$ were mixed with a Nucleofector solution plus $5 \mu \mathrm{g}$ plasmid. Following electroporation using the T-20 program, the cell suspension was immediately diluted with prewarmed growth medium and seeded into $60-\mathrm{mm}$ dishes or 12 -well plates $\left(5 \times 10^{4}\right.$ cells $\left./ \mathrm{cm}^{2}\right)$. At $48 \mathrm{~h}$ posttransfection, cells were analyzed or serum-starved overnight for treatment with gangliosides.

\section{NF-kB immunocytochemistry}

Primary astrocytes were fixed and permeabilized with ice-cold methanol for $5 \mathrm{~min}$, and washed with PBS. Cells were incubated with an anti-NF-kB p65 antibody diluted in PBS containing $1 \% \mathrm{BSA}$ for $1 \mathrm{~h}$ at $37^{\circ} \mathrm{C}$ and then with an Alexa Fluor 594-conjugated secondary antibody (Molecular Probes). VECTASHIELD mounting medium supplemented with 4',6-diamidino-2-phenylindole (DAPI; Vector Laboratories, Burlingame, CA) was used to mount samples. Images were collected on a laser scanning confocal microscope (LSM 710, Carl Zeiss Microimaging, Göttingen, Germany) equipped with C-Apochromat 40x/1.20 water immersion objective and acquired with ZEN 2009 software (Carl Zeiss Microimaging).

\section{Tubby imaging}

$\mathrm{PI}(4,5) \mathrm{P}_{2}$ was visualized by the ectopic expression of a membrane-bound transcription factor tubby that has been used as a specific probe for $\mathrm{PI}(4,5) \mathrm{P}_{2}$ (Santagata et al., 2001; Szentpetery et al., 2009). A mutant (R332H) plasmid of the full-length tubby subcloned into the eYFP-N1 vector expressing YFP at the C-terminus was provided by Andrew Tinker (University College London, UK) (Quinn et al., 2008). Primary astrocytes were transfected with the fluorescent tubby construct using Amaxa Nucleofection and seeded onto coverslips in 12 -well plates $\left(1 \times 10^{5}\right.$ cells/well). At $48 \mathrm{~h}$ posttransfection, cells were further treated with gangliosides and YFP fluorescent images in the FITC channel were obtained using an LSM 710 confocal microscope.

\section{Statistical analysis}

Band intensities of Western blots were measured using $\mathrm{NIH}$ Image J software (National Institutes of Health). Statistical significance of Western blot and real-time PCR results shown in the graphs was determined using an unpaired $t$-test (Graphpad Software, San Diego, CA) and data are presented as mean \pm SEM.

\section{Acknowledgements}

We thank Dr. Andrew Tinker for providing the tubby expression construct. This work was supported by research grants from the Korea Science and Engineering Foundation through the Chronic Inflammatory Disease Research Center at Ajou University (R13-2003-019), the Korea Research Foundation (KRF-2008-331-C00238), and Ajou University School of Medicine.

\section{References}

Akira S, Takeda K. Toll-like receptor signalling. Nat Rev Immunol 2004;4:499-511

Ariga T, McDonald MP, Yu RK. Role of ganglioside metabolism in the pathogenesis of Alzheimer's disease--a review. J Lipid Res 2008;49:1157-75

Blennow K, Davidsson P, Wallin A, Fredman P, Gottfries CG, Mansson JE, Svennerholm L. Differences in cerebrospinal fluid gangliosides between "probable Alzheimer's disease" and normal aging. Aging (Milano) 1992;4:301-6

Di Paolo G, De Camilli P. Phosphoinositides in cell regulation and membrane dynamics. Nature 2006;443:651-7

Doughman RL, Firestone AJ, Anderson RA. Phosphatidylinositol phosphate kinases put $\mathrm{PI}, 5 \mathrm{P}(2)$ in its place. $\mathrm{J}$ Membr Biol 2003;194:77-89

Farina C, Aloisi F, Meinl E. Astrocytes are active players in cerebral innate immunity. Trends Immunol 2007;28:138-45

Fitzgerald KA, Palsson-McDermott EM, Bowie AG, Jefferies CA, Mansell AS, Brady G, Brint E, Dunne A, Gray P, Harte MT, McMurray D, Smith DE, Sims JE, Bird TA, O'Neill LA. Mal (MyD88-adapter-like) is required for Toll-like receptor-4 signal transduction. Nature 2001;413:78-83

Horng T, Barton GM, Flavell RA, Medzhitov R. The adaptor molecule TIRAP provides signalling specificity for Toll-like receptors. Nature 2002;420:329-33

Ishihara H, Shibasaki Y, Kizuki N, Katagiri H, Yazaki Y, Asano T, Oka Y. Cloning of cDNAs encoding two isoforms of $68-k D a$ type I phosphatidylinositol-4-phosphate 5-kinase. J Biol Chem 1996;271:23611-4

Ishihara $\mathrm{H}$, Shibasaki Y, Kizuki N, Wada T, Yazaki Y, Asano T, Oka Y. Type I phosphatidylinositol-4-phosphate 5-kinases. Cloning of the third isoform and deletion/substitution analysis of members of this novel lipid kinase family. J Biol Chem 1998;273:8741-8

Jou I, Lee JH, Park SY, Yoon HJ, Joe EH, Park EJ. Gangliosides trigger inflammatory responses via TLR4 in brain glia. Am J Pathol 2006;168:1619-30

Jung JU, Ko K, Lee DH, Ko K, Chang KT, Choo YK. The roles of glycosphingolipids in the proliferation and neural differentiation of mouse embryonic stem cells. Exp Mol Med 2009;41:935-45

Kagan JC, Medzhitov R. Phosphoinositide-mediated adaptor recruitment controls Toll-like receptor signaling. Cell 2006;125:943-55

Li Q, Verma IM. NF-kappaB regulation in the immune system. Nat Rev Immunol 2002;2:725-34

Lund S, Christensen KV, Hedtjarn M, Mortensen AL, Hagberg $H$, Falsig J, Hasseldam $H$, Schrattenholz A, Porzgen P, Leist M. The dynamics of the LPS triggered inflammatory response of murine microglia under different culture and in vivo conditions. J Neuroimmunol 2006;180: 71-87

Mao YS, Yin HL. Regulation of the actin cytoskeleton by phosphatidylinositol 4-phosphate 5 kinases. Pflugers Arch 


\section{7;455:5-18}

Min KJ, Pyo HK, Yang MS, Ji KA, Jou I, Joe EH. Gangliosides activate microglia via protein kinase $\mathrm{C}$ and NADPH oxidase. Glia 2004;48:197-206

Padron D, Wang YJ, Yamamoto M, Yin H, Roth MG. Phosphatidylinositol phosphate 5-kinase lbeta recruits AP-2 to the plasma membrane and regulates rates of constitutive endocytosis. J Cell Biol 2003;162:693-701

Pawate S, Shen Q, Fan F, Bhat NR. Redox regulation of glial inflammatory response to lipopolysaccharide and interferongamma. J Neurosci Res 2004;77:540-51

Posse de Chaves E, Sipione S. Sphingolipids and gangliosides of the nervous system in membrane function and dysfunction. FEBS Lett 2010;584:1748-59

Pyo H, Joe E, Jung S, Lee SH, Jou I. Gangliosides activate cultured rat brain microglia. J Biol Chem 1999;274:34584-9

Quinn KV, Behe P, Tinker A. Monitoring changes in membrane phosphatidylinositol 4,5-bisphosphate in living cells using a domain from the transcription factor tubby. $\mathrm{J}$ Physiol 2008;586:2855-71

Rameh LE, Tolias KF, Duckworth BC, Cantley LC. A new pathway for synthesis of phosphatidylinositol-4,5-bisphosphate. Nature 1997;390:192-6

Sakurai $H$, Chiba H, Miyoshi $H$, Sugita T, Toriumi W. IkappaB kinases phosphorylate NF-kappaB p65 subunit on serine 536 in the transactivation domain. J Biol Chem 1999;274: 30353-6

Santagata S, Boggon TJ, Baird CL, Gomez CA, Zhao J, Shan WS, Myszka DG, Shapiro L. G-protein signaling through tubby proteins. Science 2001;292:2041-50

Sasaki J, Sasaki T, Yamazaki M, Matsuoka K, Taya C, Shitara $\mathrm{H}$, Takasuga S, Nishio M, Mizuno K, Wada T, Miyazaki H, Watanabe H, lizuka R, Kubo S, Murata S, Chiba T, Maehama T, Hamada K, Kishimoto H, Frohman MA, Tanaka K, Penninger JM, Yonekawa H, Suzuki A, Kanaho Y. Regulation of anaphylactic responses by phosphatidylinositol phos- phate kinase type I \{alpha\}. J Exp Med 2005;201:859-70

Schwab C, McGeer PL. Inflammatory aspects of Alzheimer disease and other neurodegenerative disorders. J Alzheimers Dis 2008;13:359-69

Sonnino S, Chigorno V. Ganglioside molecular species containing C18- and C20-sphingosine in mammalian nervous tissues and neuronal cell cultures. Biochim Biophys Acta 2000;1469:63-77

Suh BC, Hille B. Regulation of ion channels by phosphatidylinositol 4,5-bisphosphate. Curr Opin Neurobiol 2005; 15:370-8

Szentpetery Z, Balla A, Kim YJ, Lemmon MA, Balla T. Live cell imaging with protein domains capable of recognizing phosphatidylinositol 4,5-bisphosphate; a comparative study. BMC Cell Biol 2009;10:67

Szymanska E, Korzeniowski M, Raynal P, Sobota A, Kwiatkowska K. Contribution of PIP-5 kinase lalpha to raft-based FcgammaRIIA signaling. Exp Cell Res 2009;315: 981-95

Takenawa T, Itoh T. Phosphoinositides, key molecules for regulation of actin cytoskeletal organization and membrane traffic from the plasma membrane. Biochim Biophys Acta 2001;1533:190-206

Teismann P, Schulz JB. Cellular pathology of Parkinson's disease: astrocytes, microglia and inflammation. Cell Tissue Res 2004;318:149-61

van den Bout I, Divecha N. PIP5K-driven Ptdlns(4,5)P2 synthesis: regulation and cellular functions. J Cell Sci 2009;122:3837-50

Yamamoto $M$, Hilgemann $D H$, Feng $S$, Bito $H$, Ishihara $H$, Shibasaki Y, Yin HL. Phosphatidylinositol 4,5-bisphosphate induces actin stress-fiber formation and inhibits membrane ruffling in CV1 cells. J Cell Biol 2001;152:867-76

Yang F, Tang E, Guan K, Wang CY. IKK beta plays an essential role in the phosphorylation of RelA/p65 on serine 536 induced by lipopolysaccharide. J Immunol 2003;170: 5630-5 